\title{
ALMOST-DEDEKIND RINGS
}

\author{
by E. W. JOHNSON
}

(Received 15 September, 1992)

Throughout we assume all rings are commutative with identity. We denote the lattice of ideals of a ring $R$ by $L(R)$, and we denote by $L(R)^{*}$ the subposet $L(R)-R$.

A classical result of commutative ring theory is the characterization of a Dedekind domain as an integral domain $R$ in which every element of $L(R)^{*}$ is a product of prime ideals (see Mori [5] for a history). This result has been generalized in a number of ways. In particular, rings which are not necessarily domains but which otherwise satisfy the hypotheses (i.e. general ZPI-rings) have been widely studied (see, for example, Gilmer [3]), as have rings in which only the principal ideals are assumed to satisfy the hypothesis (i.e. $\pi$-rings).

General ZPI-rings and $\pi$-rings can both be thought of as "almost Dedekind". In both cases, one gets a representation as the finite direct product of integral domains of the same type (Dedekind domains in the first case, $\pi$-domains in the second case) and quotients of discrete (rank one) valuation rings (i.e. special principal ideal rings-or SPIRS as they have come to be called).

Note that ZPI-rings are rings in which every ideal in $L(R)^{*}$ satisfies the "product of prime ideals" condition, whereas only the principal ideals of a $\pi$-ring are assumed to satisfy this condition. This naturally raises consideration of rings in which every ideal of $L(R)^{*}$ generated by $n$ elements is a product of prime ideals. Any UFD is a $\pi$-ring; so a $\pi$-ring need not be a general ZPI-ring. In this regard, Levitz $[4,5]$ has obtained the very interesting result that $\pi$-rings are the single exception. If every doubly generated ideal in $L(R)^{*}$ is the product of prime ideals, then every ideal in $L(R)^{*}$ is.

Butts and Gilmer [3] have characterized ZPI-rings in a somewhat different manner. They have shown that ZPI-rings are characterized by the property that every ideal in $L(R)^{*}$ is a finite intersection of powers of prime ideals.

In this paper, we obtain the analogue of Levitz's theorem for the Butts-Gilmer characterization of general ZPI-rings. That is, we show that, once again, two elements suffice: if $R$ is a ring in which every double generated ideal in $L(R)^{*}$ is the intersection of powers of prime ideals, then every ideal in $L(R)^{*}$ is.

For convenience, we will say that a ring $R$ satisfies "Property D" if every doubly generated ideal in $L(R)^{*}$ is the intersection of powers of prime ideals.

We begin with a simple but useful observation.

Lemma 1. Let $(R, M)$ be a quasi-local ring satisfying Property $D$. If $x, y \in M$ then there are only a finite number of primes minimal over $(x, y)$.

Proof. $(x, y)$ is the finite intersection of powers of prime ideals, say $(x, y)=\bigcap_{i=1}^{n} P_{i}^{e_{i}}$. Then any prime minimal over $(x, y)$ is one of the primes $P_{i}$.

We also note the following.

Lemma 2. If $R$ satisfies Property $D$ and if $S$ is a multiplicatively closed subset of $R$, then $R_{S}$ satisfies Property $D$.

Proof. $\left(a / s_{1}, b / s\right) R_{S}=(a, b) R_{S}$. 
The following appears in a different form in [1]. It reduces the problem for quasi-local rings to two cases.

Lemma 3. Let $(R, M)$ be a quasi-local ring satisfying Property $D$. Then either $M$ is principal or $M=M^{2}$.

Proof. Assume $M \neq M^{2}$. Choose $x \in M-M^{2}$ and let $y$ be any element of $M$. Then $\left(x, y^{2}\right)$ is the intersection of prime powers, say $\left(x, y^{2}\right)=\bigcap_{i=1}^{s} P_{i}^{e_{i}}$. As $x \notin M^{2}$, it follows that $\left(x, y^{2}\right)=\bigcap_{i=1}^{s} P_{i}$. But $y^{2} \in P_{i}$ implies $y \in P_{i} ;$ so also $(x, y)=\bigcap_{i=1}^{s} P_{i}=\left(x, y^{2}\right)$. By Nakayama's lemma, it follows that $y \in(x)$. By the choice of $y$, it follows that $M=(x)$.

The following addresses the quasi-local case with $M$ principal.

Lemma 4. Let $(R, M)$ be a quasi-local ring satisfying Property $D$. If $M$ is a principal ideal of $R$, then every nonzero ideal of $R$ is a power of $M$.

Proof. If $M$ is a minimal prime of $R$, then $M$ is the only prime ideal of $R$. It follows that every nonzero principal ideal of $R$ is a power of $M$, and hence that every nonzero ideal of $R$ is a power of $M$.

Hence, assume $M=(x)$ is not a minimal prime ideal of $R$. If $P$ is any prime ideal of $R$, then $P=P \cap(x)=(P: x) x=P M$; so $P \subseteq \bigcap_{i=1}^{\infty} M^{n}$. Set $P_{0}=\bigcap_{i=1}^{\infty} M^{n}$. Note that $M^{n}=$ $M^{n+1}$ would imply $M^{n}=0$ (by Nakayama's lemma), which would, in turn, contradict the assumption that $M$ is not minimal. Hence $M^{n} \neq M^{n+1}$ for all $n$. Then $x \in M^{r}-M^{r+1}$ and $y \in M^{s}-M^{s+1}$ imply $(x)=M^{r}$ and $(y)=M^{s}$, whence $(x y)=(x)(y)=M^{r+s}$. It follows that $x y \notin M^{r+s+1}$, and hence that that $P_{0}$ is prime. Note that $P_{0}$ contains all prime ideals of $R$ different from $M$.

If $P_{0}$ is a minimal prime ideal of $R$, then either $P_{0}=0$, in which case $R$ has exactly two prime ideals and both are principal, or else $P_{0} \neq 0$ but $P_{0}^{n}=0$ for some $n \geq 2$. In this case, choose $z \in P_{0}-P_{0}^{2}$. Then $(z)$ is of the form $P_{0}^{i}$, so $(z)=P_{0}$. Once again $R$ has exactly two prime ideals and both are principal.

Hence if $P_{0}$ is a minimal prime of $R$, then $(R, M)$ is a local Noetherian domain with $M$ a principal ideal. If $A \in L(R)^{*}$ with $A \subseteq M^{n}$ and $A \subseteq M^{n+1}$, then $A=A \cap M^{n}=$ $\left(A: M^{n}\right) M^{n}$ with $\left(A: M^{n}\right) \subseteq M ;$ so $\left(A: M^{n}\right)=R$ and $A=M^{n}$.

Now, assume $P_{0}$ is not a minimal prime. By Lemma 1 , the number of minimal primes is finite. Choose $z \in P_{0}$ outside of all minimal primes of $R$. Let $Q$ be a prime minimal over $(z)$. Then $z R_{Q}$ is a power of $Q R_{Q}$ and $z R_{Q} \neq z^{2} R_{Q}$, so $Q R_{Q} \neq Q^{2} R_{Q}$. Choose $y \in Q-\left(Q^{2}\right)_{Q}$. Then $(z, y)$ is the intersection of powers of distinct primes, say $(z, y)=\bigcap_{i=1}^{t} P_{i}^{e_{i}}$, with (say) $Q=P_{1}$. Then $e_{1}=1$ and $(z, y) R_{Q}=Q R_{Q}=\left(z^{2}, y\right) R_{Q}$. It follows that $Q R_{Q}=y R_{Q}$.

We now show that the powers of $Q$ are primary. Hence, assume $r s \in Q^{n}$ and $s \notin Q$. Then $r \in Q$ and, as above, $\left(r, y^{n}\right)$ is an intersection of powers of distinct primes, $\left(r, y^{n}\right)=\bigcap_{i=1}^{1} P_{i}^{e_{i}}$, with (say) $Q=P_{1}$. Then $\left(r, y^{n}\right) R_{Q}=Q^{e_{1}}$. Since also $\left(r s, y^{n}\right) R_{Q}=$ 
$\left(r, y^{n}\right) R_{Q}=Q^{e_{1}} R_{Q}$, it follows that $e_{1}=n$, whence $\left(r, y^{n}\right) \subseteq Q^{n}=P_{1}^{e_{1}}$. Hence $r \in Q^{n}$, and $Q^{n}$ is $Q$-primary.

The ideal $(y)$ also is an intersection of powers of distinct primes, say $(y)=\bigcap_{i=1}^{1} Q_{i}^{e_{i}}$. Here, it can be assumed that $Q_{i} \subseteq P_{0}, i=1, \ldots, t$. For $i=1, \ldots, t$, let $E_{i}$ be a prime ideal which is both minimal over $(y)$ and contained in $Q_{i}$.

By the above, the powers of $E_{i}$ are primary for $E_{i}$. From $0=(0: M) M \subseteq E_{i}^{e_{i}}$ and $M \nsubseteq E_{i}$, it follows that $(0: M) \subseteq E_{i}^{e_{i}}$ for all $i=1, \ldots, t$. Then $(0: M) \subseteq Q_{i}^{e_{i}}, i=1, \ldots, t$, as well. From this and the assumption that $M$ is principal, we have $M Q^{i}=Q^{i}, i=1, \ldots, t$ and $M(y)=M \bigcap_{i=1}^{1} Q_{i}^{e_{1}}=\bigcap_{i=1}^{t} M Q_{i}^{e_{i}}=\bigcap_{i=1}^{1} Q_{i}^{e_{i}}=(y)$. By Nakayama's lemma, $y=0$, which contradicts the choice of $y$ and completes the proof.

The following addresses the quasi-local case with $M=M^{2}$.

Lemma 5. Let $(R, M)$ be a quasi-local ring satisfying Property $D$ in which $M=M^{2}$. Then every ideal of $R$ is a power of $M$.

Proof. Assume $M$ is not a minimal prime of $R$. Let $Q_{i}, i=1, \ldots, t$, be the minimal primes of $R$. Choose $x \in M-\bigcup_{i=1}^{t} Q_{i}$, and let $P_{i}, i=1, \ldots, r$, be the primes minimal over $(x)$. If $M \in\left\{P_{i}\right\}, i=1, \ldots, t$, then $t=1$ and $(x)$ is a power of $M$. It follows that $(x)=M$, and hence that $M=0$. Hence, $M \neq P_{i}, i=1, \ldots, r$. Note that any rank one prime containing $x$ is minimal over $(x)$.

Choose $y \in M-\bigcup_{i=1}^{r} P_{i}$, so $(x, y)$ is not contained in any rank zero prime or any rank one prime. Let $Q$ be minimal over $(x, y)$. By passing to $R_{Q}$, we may assume that $Q$ is maximal and that $(x, y)$ is a power of $Q$. Since $(x, y) \neq(x, y)^{2}$, it follows that $Q R_{Q} \neq Q^{2} R_{Q}$, and hence (by Lemma 3) that $Q R_{Q}$ is principal in $R_{Q}$. By the previous lemma, $Q$ has rank at most one, which contradicts the choice of $Q$.

Hence $M$ is minimal over 0 , and necessarily is the only prime ideal of $R$. It follows that every principal ideal of $R$ is a power of $M$, and hence that every ideal of $R$ is a power of $M$.

We now globalize.

Theorem 6. Let $R$ be a ring satisfying Property $D$. Then $R$ is the finite direct product of special principal ideal rings, fields and (one-dimensional) Dedekind domains.

Proof. Let $0=\bigcap_{i=1}^{n} P_{i}^{e_{i}}$ be a representation of 0 as an intersection of powers of distinct primes. In this case, it can be assumed that each prime $P_{i}$ is minimal over 0 . By the previous lemmas, each maximal ideal of $R$ contains exactly one minimal ideal of $R$, so the primes $P_{1}, \ldots, P_{n}$ are comaximal. It follows that $R=R_{1} \times \cdots \times R_{n}$, where each $R_{i}$ is isomorphic to $R / P_{i}^{e_{i}}$. Note that each factor $R_{i}$ inherits Property $\mathrm{D}$ from $R$. By passage to one of the factors, we can assume that $R$ has a unique minimal prime $P$.

If $P$ is maximal, then $R$ is local, and $R$ is either a field or a special principal ideal ring. Hence, assume $P$ is not maximal. By the previous lemma, it follows for each 
maximal ideal $M$ that $M \neq M^{2}$ and that $R_{M}$ is a DVR. Hence $R$ is an integral domain and every nonzero prime ideal of $R$ is maximal. It follows from Property D that every doubly generated ideal of $R$ is the product of prime ideals.

The result now follows from Levitz's theorem.

THEOREM 7. Let $R$ be a ring. Then $R$ is a general ZPI-ring if, and only if, every doubly generated ideal in $L(R)^{*}$ is the finite intersection of prime powers.

Proof. This is immediate from Theorem 6.

\section{REFERENCES}

1. D. D. Anderson and E. W. Johnson, A new characterization of Dedekind domains, Glasgow Math. J. 28 (1986), 237-239.

2. H. S. Butts and R. W. Gilmer Jr., Primary ideals and prime power ideals, Canad. J. Math. 18 (1966), 1183-1195.

3. R. Gilmer, Multiplicative ideal theory (Marcel Decker, 1972).

4. K. B. Levitz, A characterization of general Z.P.I.-rings, Proc. Amer. Math. Soc. 32 (1972), $376-380$.

5. K. B. Levitz, A characterization of general Z.P.I.-rings II, Pacific J. Math. 42 (1972), 147-151.

6. S. Mori, Allgemeine Z.P.I.-Ringe, J. Sci. Hiroshima Univ. Ser. A. 10 (1940), 117-136.

THE UNIVERSITY OF IOWA

IOWA CiTY, IOWA 